\title{
Children's resistance to extinction: Two tests of the discrimination hypothesis
}

\author{
KENNETH L. WITTE \\ University of Arkansas, Fayetteville, Arkansas 72701
}

\begin{abstract}
Experiment 1 investigated the effects of three reinforcement schedules, $100 \%, 50 \%$ alternating, and $50 \%$ random, upon kindergarten and third-grade children's resistance to extinction in a free operant task involving a leverpulling response on a slot machine. The $50 \%$ random condition produced the greatest amount of resistance to extinction, while the $100 \%$ condition produced the least. The results were interpreted as consistent with the accounting of the partial reinforcement extinction effect by the discrimination hypothesis. Experiment 2 used the same apparatus as used in Experiment 1 with four groups of first-grade children. The children were trained with either $50 \%$ random or $100 \%$ reinforcement or with one type of reinforcement schedule followed by the other, and were then given extinction. There were no significant differences in resistance to extinction among the three groups of children experiencing partial reinforcement, and each of the three groups exhibited more resistance to extinction than the group of children receiving $100 \%$ reinforcement. These results were seen as in disagreement with a simple version of the discrimination hypothesis, although not necessarily with a revised and elaborated form of the hypothesis, such as that proposed by Capaldi (1967).
\end{abstract}

The effects of various schedules of reinforcement upon resistance to extinction in animals have been studied extensively, and the partial reinforcement extinction effect (PREE) is a reliable phenomenon. However, Reese and Lipsitt (1970) indicate that the effects of different reinforcement schedules upon children's responses have not been studied extensively, and that the relation between schedules of reinforcement and resistance to extinction in studies using either free operant or discrete-trial tasks is not altogether clear.

Given the relative sparsity of research with children and the inconsistency of the available findings, it is obvious that systematic research is needed. Thus, one goal of this research was to provide additional data concerned with children's resistance to extinction. A second goal was to obtain evidence relevant to the process or processes responsible for the PREE. One process undoubtedly important is the ease with which the child can discriminate between acquisition and extinction conditions. According to the discrimination hypothesis (Gonzalez \& Bitterman, 1964), the rate of extinction is inversely related to the degree of similarity between acquisition and extinction conditions. Thus, the PREE occurs because it is harder for an organism trained with partial reinforcement to discriminate between acquisition and extinction than it is for an organism trained with continuous reinforcement.

This research was supported, in part, by a Research Reserve Fund grant from the University of Arkansas. Reprint requests should be sent to Kenneth L. Witte, Department of Psychology, University of Arkansas, Fayetteville, Arkansas 72701. This paper is sponsored by Joel S. Freund, who takes full editorial responsibility for its contents.

\section{EXPERIMENT 1}

This experiment examined children's extinction as related to three conditions of acquisition: continuous reinforcement, $50 \%$ alternating reinforcement, and $50 \%$ random reinforcement. The discrimination between acquisition and extinction should be relatively easy for children in the first condition and in the second condition if they learn the alternating sequence. The discrimination should be the hardest for the children in the random condition. Previous research with animals and with adults (see Hulse, Deese, \& Egeth, 1975 for a review) has found that random reinforcement produces a greater PREE than does alternating reinforcement. This study tested children from two grades $(K, 3)$ in order to examine for developmental trends. If increasing mental age results in an increasing ability to discriminate changes in stimulus conditions, then, according to the discrimination hypothesis, the older children should extinguish faster than the younger children.

\footnotetext{
Method

Subjects. The subjects were 42 kindergarten children (18 boys, 24 girls) and 51 third graders ( 33 boys, 18 girls). All subjects were white and most came from middle- to lowermiddle-class families.

Apparatus. The apparatus was a modified slot machine. The three payoff display windows centered in the front face of the machine were covered in order to avoid distracting the children. A lever was mounted on the right side of the machine; pulling the lever rotated a payoff wheel one position forward and also activated a response counter. Quarter-sized slugs were dispensed into a small cup at the lower right front of the machine. Additional materials included a variety of inexpensive (20-cent) toys.
}

Design. A 2 by 2 by 3 factorial design involved two grades 
$(K, 3)$, two sexes (male, female), and three reinforcement schedules $(100 \%, 50 \%$ alternating, $50 \%$ random). Children within a given grade-sex condition were assigned to a reinforcement group via a block randomization procedure. Four different $50 \%$ random reinforcement schedules were constructed, and children within this condition were assigned one of the four schedules via a block randomization method. At the kindergarten level there were 6 boys and 8 girls in each reinforcement condition, while at the third-grade level the numbers were 11 and 6 , respectively.

Procedure. Children were tested individually. A child was told that he/she was going to play the "money machine game," asked to pull the lever of the slot machine, and reinforced for this initial response. The child was shown the reward slug, told it was "play money," and told that if he/she obtained enough money, he/she could exchange it for a toy of his/her choice. Each child was then asked to pull the lever again. This response was nonreinforced and all children were told they might not always receive money for pulling the lever. Finally, all children were instructed that they could play the game as long as they wished.

Each child was then given 16 acquisition trials followed, without any ostensible break, by extinction. To any questions or comments by the child during the game the experimenter replied, "Remember, you can play the game as long as you like. Just tell me when you want to stop." Extinction continued until the child asked to stop, or after 102 extinction trials, ${ }^{1}$ whichever occurred first.

\section{Results}

A 2 (age) by 2 (sex) by 3 (reinforcement schedule) analysis of variance of transformed trials to extinction scores $(\log \mathrm{X})$ resulted in one reliable effect, that for reinforcement condition $[\mathrm{F}(2,81)=8.11, \mathrm{p}<.001]$. A Duncan's multiple-range test indicated that the $50 \%$ random condition produced reliably greater resistance to extinction than either the continuous $(p<.005)$ or $50 \%$ alternating $(p<.05)$ conditions, while the difference between the latter two conditions was marginally significant $(\mathrm{p}<.10)$.

\section{Discussion}

The finding that random partial reinforcement produced greater resistance to extinction than did continuous reinforcement is consistent with the results of earlier developmental studies (Kass, 1962; Kass \& Wilson, 1966). In addition, the difference between the two partial reinforcement groups is comparable to that obtained in previous research with animals and with adults. The results are also generally consistent with the discrimination hypothesis in that trials to extinction were greatest for the random partial reinforcement group and fewest for the continuous reinforcement group. Somewhat surprisingly, the alternating partial and continuous reinforcement groups differed, albeit not at an acceptable level of significance. It was expected that the discrimination between acquisition and extinction conditions would be relatively easy for children in both groups since they experienced a regular sequence of reinforcement; therefore, trials to extinction for the two groups should have been comparable. However, it is possible that not all children in the $50 \%$ alternation condition learned the reinforcement sequence. Sixteen acquisition trials were used in the present study; Bogartz (1966, Experiment 3) found that $60 \%$ of his 4- and 5-year-old subjects learned an alternation sequence within 16 trials. If some children in the $50 \%$ alternation condition did not learn the alternation sequence, then the functional reinforcement sequence for them would have been a $50 \%$ random one, thereby increasing their resistance to extinction and inflating the mean score for the group as a whole.
Somewhat surprisingly, the older children did not extinguish faster than the younger children. At least three factors could have operated to counteract the expected age difference in extinction. First, pulling the lever required a good deal of physical strength. As a result, difficulty in pulling the lever may have resulted in the smaller, and therefore younger, children quitting the game sooner than the larger, older children. Second, many of the third graders may have preferred to stay and play the game, even if they were reasonably sure that no more money was to be obtained, rather than return to class and work assignments. A number of third graders, but no younger children, spontaneously commented to this effect. Finally, as noted by Kass (1962), the attention span of the younger children may have been less than that of the older children.

\section{EXPERIMENT 2}

Although the results of the first study are generally consistent with the discrimination hypothesis, research using organisms other than children indicates that this hypothesis must be modified if it is to satisfactorily account for the PREE. One line of research particularly damaging to a simple discrimination hypothesis is that in which partially reinforced animals are given a series of continuously reinforced trials prior to extinction (see Hulse et al., 1975 for a review). According to the discrimination hypothesis, subjects given interpolated trials involving continuous reinforcement should extinguish at the same rate as subjects receiving continuous reinforcement throughout. This is the case because, on the trials prior to extinction, the pattern of continuous reinforcement then in effect should make it easy for the subjects to discriminate the change to the consistent nonreinforcement of extinction. However, the available research indicates that the effects of partial reinforcement are sustained through a series of continuously reinforced trials, in that subjects receiving the interpolated training show more resistance to extinction than continuously reinforced subjects. This study examined the effects of such interpolated training upon children's resistance to extinction in order to provide further data pertaining to the adequacy of the discrimination hypothesis in accounting for the PREE.

\section{Method}

Subjects. The subjects were 40 first-grade children, 20 of each sex, attending one of the schools involved in Experiment 1.

Apparatus. The apparatus used was that described in Experiment 1. An additional piece of equipment was a large, clear plastic container. Slugs won by the children were placed in this container rather than being left in the reward cup of the slot machine.

Design. A 2 by 2 by 2 factorial design involved the variables of sex, reinforcement schedule during the first half of acquisition $(100 \%, 50 \%$ random), and reinforcement schedule during the second half of acquisition (100\%, $50 \%$ random). Children of each sex were assigned to one of the four acquisition groups via a block randomization procedure. Four different eighttrial sequences of $50 \%$ random reinforcement were constructed, and the sequence(s) used with a given child was (were) randomly determined.

Procedure. The procedure was essentially the same as that used in Experiment 1. The children were given two practice trials; the first was rewarded, while the second was not. Then 
all children received 16 acquisition trials followed by extinction. ${ }^{2}$ Extinction continued until the child asked to stop, or after 102 extinction trials, whichever occurred first.

\section{Results}

Mean trials to extinction for Groups 100-100, $50-100,100-50$, and $50-50$ were $16.20(\mathrm{SD}=22.43)$, $43.60(\mathrm{SD}=31.71), 42.90(\mathrm{SD}=30.59)$, and 41.20 $(S D=34.98)$, respectively. The raw scores were transformed $(\log X)$ and used in a 2 (sex) by 2 (first-half reinforcement schedule) by 2 (second-half reinforcement schedule) analysis of variance. One reliable statistic was obtained, the interaction between reinforcement schedules for the two halves of acquisition $[F(1,23)=5.66$, $\mathrm{p}<.025]$. Resistance to extinction was comparable for the three groups experiencing partial reinforcement, and each of these groups differed from the group experiencing only continuous reinforcement.

\section{Discussion}

The results of the present study are inconsistent with the discrimination hypothesis in its simplest form. Specifically, the finding that the children receiving $100 \%$ reinforcement after first receiving partial reinforcement extinguished at the same rate as the other two groups experiencing partial reinforcement, and slower than the group experiencing only continuous reinforcement, is most damaging to the hypothesis. This is because the pattern of continuous reinforcement in effect during the latter half of acquisition should have made it relatively easy for the children to discriminate the change to the consistent nonreinforcement characteristic of extinction.

The finding that the PREE was obtained for both groups receiving interpolated training is consistent with the animal literature which indicates that the PREE is obtained whether continuously reinforced trials are given before or after a partial schedule. However, there is conflict regarding which condition produces the greater amount of resistance to extinction (Robbins, 1971). Nonetheless, the important issue is not whether the two groups receiving interpolated training differ from each other, but whether the group receiving continuous reinforcement following random reinforcement differs from the continuously reinforced group. As indicated earlier, this finding was obtained and is inconsistent with the discrimination hypothesis.

Although the present results, plus those obtained earlier with animal and adult human subjects, are inconsistent with the discrimination hypothesis, it is possible that the hypothesis could be appropriately modified to account for these data (Hulse et al., 1975). In this regard, Capaldi's (1967) sequential theory is, in part, a sophisticated elaboration and refinement of the discrimination hypothesis (Hilgard \& Bower, 1975, p. 567) and is viewed by many (e.g., Robbins, 1971) as the best current explanation of the PREE. In this regard, Capaldi's theory has experienced some initial success in accounting for the PREE in children (Miyao \& Meyers, 1973).

\section{REFERENCES}

BogARTz, R. S., Variables influencing alternation prediction by preschool children: I. Previous recurrent, dependent, and repetitive sequences. Journal of Experimental Child Psychology, 1966, 3, 40-56.

CAPALDI, E. J. A sequential hypothesis of instrumental learning. In K. W. Spence \& J. T. Spence (Eds.), The psychology of learning and motivation (Vol. 1). New York: Academic Press, 1967.

Gonzalez, R. C., \& Bitterman, M. E. Resistance to extinction in the rat as a function of percentage and distribution of reinforcement. Journal of Comparative and Physiological Psychology, 1964, 58, 258-263.

HilgaRD, E. R., \& Bower, G. H. Theories of learning (4th ed.). Englewood Cliffs, N.J: Prentice-Hall, 1975.

Hulse, S. H., Deese, J., \& Egeth, H. The psychology of learning (4th ed.). New York: McGraw-Hill, 1975.

Kass, N. Resistance to extinction as a function of age and schedules of reinforcement. Journal of Experimental Psychology, 1962, 64, 249-252.

Kass, N., \& Wilson, H. Resistance to extinction as a function of percentage of reinforcement, number of training trials, and conditioned reinforcement. Journal of Experimental Psychology, 1966, 71, 355-357.

Miyao, G., \& MeYers, L. S. A sequential analysis of the partial reinforcement extinction effect in children. Memory \& Cognition, 1973, 1, 143-148.

ReEse, H. W., \& LipsitT, L. P. Experimental child psychology. New York: Academic Press, 1970.

Robrins, D. Partial reinforcement: A selective review of the alleyway literature since 1960. Psychological Bulletin, 1971, 76. $415-431$.

\section{NOTES}

1. A maximum of 120 trials could be given with the slot machine. Since 18 trials, including the 2 practice trials, were given during acquisition, no more than 102 extinction trials could be given.

2. The number of acquisition trials in this experiment is the same as that given in Experiment 1. Since it was indicated there that 16 trials were possibly not enough to produce the exact pattern of expected results, one may wonder why more trials were not given in Experiment 2. In Experiment 1 the issue involved whether 16 trials were enough for the alternation sequence to be learned; there is no alternation sequence in Experiment 2. The number of trials is critical here only in regard to whether the eight trials of partial reinforcement experienced by the two groups receiving interpolated training are enough to produce the PREE. Earlier developmental research by Kass and Wilson (1966) indicated that eight trials would suffice. These authors, using a simulated slot machine apparatus, examined the magnitude of the PREE after 3, 9, 21, 45, and 60 acquisition trials for children receiving either variable (33-1/3\%) or continuous reinforcement. A reliable PREE was obtained and this effect was independent of the number of acquisition trials.

(Received for publication November 13, 1976.) 